\title{
Ubicación de dispositivos móviles en ambientes interiores por medio de análisis de radiación de redes WiFi y deformaciones de campo magnético
}

\author{
Locating mobile devices in indoor environments by analysis of WiFi network \\ radiation and magnetic field distortions \\ Carlos A. Gómez R. ${ }^{1 *} \quad$ Luis F. Pedraza ${ }^{2}$ \\ Recibido 2 de septiembre de 2016, aceptado 31 de mayo de 2017 \\ Received: September 2, 2016 Accepted: May 31, 2017
}

\begin{abstract}
RESUMEN
La localización dentro de ambientes de interiores basada en dispositivos móviles modernos es un área actual de investigación, dado que constituye una fuente de información indispensable para el desarrollo de servicios según el contexto local del usuario. Es importante el estudio de las diferentes variables que permiten obtener información captable por teléfonos inteligentes sobre el entorno espacial del usuario, así como las fuentes de errores de esas informaciones y la forma de mitigarlos. Este trabajo presenta un modelo de uso de la radiación de las redes WiFi y la deformación del campo magnético terrestre en estructuras cerradas en interiores como fuente de información del contexto de la ubicación de un dispositivo móvil, donde todas las labores de captura, procesamiento y análisis de datos sean hechas por un teléfono inteligente, sin la necesidad de asistencia externa y con la posibilidad futura de crear un sistema de navegación autónoma en interiores. Se demuestra cómo las huellas de campos magnéticos, debido a perturbaciones del campo magnético terrestre en interiores, constituyen una fuente de rápido análisis para dispositivos móviles pero disminuyen la exactitud lograda por análisis más complejos basados exclusivamente en la radiación de redes WiFi.
\end{abstract}

Palabras clave: Localización, interiores, WiFi, campo magnético, modelo.

\begin{abstract}
The indoor location based on modern mobile devices, is a current area of research because it constitutes an indispensable source of information for the development of services based on the local context of the user. It is important to study the different variables which can obtain information about the space environment of the user using smartphones, as well as the sources of errors and how to mitigate them. This paper presents a model using WiFi radiation and deformation of the earth's magnetic field in indoors structures, as a source of information of the context of the location of a mobile device, where all the work of capture, processing and analysis data are made by a smart phone, without the need for external assistance and the future possibility of creating an indoor and autonomous navigation system. It is demonstrated how magnetic field traces due to disturbances of the terrestrial magnetic field in indoor are a source of rapid analysis for mobile devices, but they diminish the accuracy achieved by more complex analyzes based exclusively on the radiation of WiFi networks.
\end{abstract}

Keywords: Location, indoor, WiFi, magnetic field, model.

1 Universitaria Agustiniana. Avenida Ciudad de Cali No 11b-95, Bogotá D.C., Colombia.

2 Universidad Distrital "Francisco José de Caldas". Carrera 7 No 40B-53. Bogotá D.C., Colombia.

E-mail: lfpedrazam@udistrital.edu.co

* Autor de correspondencia: E-mail: carlos.gomezr@uniagustiniana.edu.co 


\section{INTRODUCCIÓN}

Analizar cómo se radian las redes WiFi para determinar la ubicación de un dispositivo, y predecir sus movimientos ha sido uno de los principales enfoques de trabajos anteriores sobre localización en interiores, dada la masificación de las redes WiFi y a la facilidad de encontrar dispositivos clientes con tarjetas WiFi. Existen tres técnicas para determinar la ubicación de un usuario: el ángulo en que la señal llega (DOA) para estimar la localización relativa de un dispositivo respecto al otro, el tiempo en que la señal llega (TOA) con la que se puede estimar el tiempo que tomó en propagarse la señal y así estimar la distancia que separa un dispositivo y otro $\mathrm{y}$, finalmente, la potencia con la que la señal es recibida (RSSI) que puede servir para estimar la distancia que separa dos dispositivos, o las características de propagación de la radiofrecuencia en un espacio [1]. Aunque el enfoque de uso de redes WiFi ha imperado en las investigaciones anteriores se ha evidenciado la necesidad de incluir nuevas variables que refuercen el proceso de localización en interiores; variables como sensores de orientación, aplicaciones de radio-frecuencia, aplicaciones ópticas, etc.

El artículo inicialmente presenta los referentes de investigación sobre las técnicas de localización de dispositivos electrónicos en interiores. Luego, se aborda la temática central en la que se parte de un método de localización por radiación de redes WiFi y se plantea un nuevo modelo que vincula el estudio de las variaciones del campo magnético en interiores, para tener alternativas en el procesamiento de datos. Posteriormente, se exhiben los datos obtenidos en los experimentos realizados, y se discute la viabilidad del modelo desarrollado para la localización de un dispositivo móvil en interiores, bajo las condiciones allí explicadas. Finalmente, se muestran las conclusiones obtenidas.

\section{ESTADO DEL ARTE}

Los ambientes de exteriores se caracterizan por tener clara línea de vista para la radiación sin obstáculo en grandes áreas, situación llamada LOS, mientras que los ambientes de interiores se caracterizan por tener gran cantidad de obstáculos para la comunicación directa entre los equipos, constituyendo escenarios de no línea de vista
(NLOS) [2] donde la cantidad de variables no permite una efectiva predicción de la radiación de las ondas electromagnéticas, y es mucho más difícil e imprecisa la interpretación de los datos relativos a la localización que llegan al dispositivo móvil. En estos ambientes NLOS los enfoques de localización por DOA y TOA, comparadas con la RSSI, presentan características de desempeño muy inferior, y con errores muy grandes. Los escenarios NLOS llevan a efectos inesperados en relación a la función potencia-distancia que pueden ser reducidos aplicando técnicas de filtrado a las mediciones. Así, muchas de las técnicas de identificación, mitigación y localización en escenarios NLOS han sido diseñadas basándose en la variable de RSSI. [3].

Las técnicas de triangulación dependen del conocimiento exacto de la distancia entre un punto desconocido y por lo menos tres puntos de referencia; sin embargo, las técnicas de análisis de potencia RSSI no pueden determinar dichas distancias precisas, ya que los diferentes elementos presentes en los obstáculos, sus materiales de construcción y fenómenos físicos como la multitrayectoria de las señales transmitidas no dejan establecer una relación directa entre la pérdida de potencia RSSI en función de la distancia recorrida en un espacio de interiores, como pasa en un escenario de exteriores [4].

Debido a lo anterior, algunos equipos de trabajo interesados en la localización de dispositivos en interiores, como el equipo de [5] en su proyecto RADAR han propuesto una metodología llamada fingerprinting, en donde el proceso de localización se hace en dos etapas: calibración y localización. En la calibración se construye una base de datos que representa las mediciones típicas de RSSI de cada red inalámbrica detectada en un punto del espacio de interiores, distinguida por su SSID (service set ID) y la dirección MAC de su Punto de Acceso (AP). Esa calibración proporciona un mapa de potencias, ya que se debe recorrer todo el espacio y caracterizar las potencias de RSSI de todas las redes WiFi.

La etapa de localización se llama al momento en el que un dispositivo que quiera localizarse, hace una caracterización de la potencia RSSI de todas las redes WiFi incidentes en su punto. Con esa 
información se realiza un cálculo comparativo entre el mapa de potencias calibrado y la medición del usuario. A partir de esa comparación se realiza la localización del dispositivo [6].

Algunos trabajos como [7] y [8] realizan un trabajo experimental que se basa en el muestreo masivo de potencia RSSI en toda el área de un espacio de interiores y aplicar técnicas de filtrado probabilísticos como umbrales de desviaciones estándar, para medir la dispersión estadística de las muestras, descartando mediciones inestables que son las fuentes comunes de error. El criterio usado por ellos es fijar un umbral superior en el análisis de desviación estándar hecho sobre las mediciones de RSSI de cada una de las señales (SSID) de Puntos de Acceso (AP) medibles en cada ubicación del mapa de RSSI para la calibración del fingerprinting. Este criterio se usa al realizar un muestreo de señales WiFi presentes en un punto de medición, del mapa de potencias RSSI. Tomando las muestras asociadas a un solo AP se estudia la dispersión de dichas muestras. Usando el criterio de [7] se descartan las muestras que tengan una alta dispersión, ya que se asume que corresponden a fuentes inestables. Este mismo criterio se aplica a cada señal WiFi presente en cada punto del espacio a calibrar, lo que termina generando un completo mapa de potencias RSSI.

En la etapa de localización propuesta por [7] se aplica la misma metodología y criterio explicado para la obtención del mapa de potencias RSSI, la diferencia es que solo se toman muestras en un solo punto del espacio, el punto en el que en un instante de tiempo se encuentra un dispositivo que necesita ser localizado. Se crea entonces un vector de promedios de potencias RSSI de las señales WiFi que hayan superado los criterios del filtro estadístico planteados más adelante en las ecuaciones 1 y 2 , llamado vector de localización. La metodología de fingerprinting exige realizar entonces una comparación entre el vector de localización y el mapa de potencias RSSI, esto se hace por medio del cálculo de distancias euclidianas expuestas en [8].

La metodología propuesta por [7] logra localizar un dispositivo móvil; sin embargo, se estudia la forma de minimizar los errores y mejorar la exactitud de la localización. En las secciones de diseño y resultados presenta un estudio de caso para probar la aplicación de esa propuesta.

En [9] se publica un trabajo donde presenta un modelo que logra mejorar la distancia de error en la localización de un teléfono inteligente, basado en el estudio de un modelo previo que usaba exclusivamente el análisis de fingerprinting de RSSI para WiFi. Usar este modelo permite hacer localización basada exclusivamente en las capacidades de procesamiento de un teléfono inteligente para grandes cantidades de datos.

En [10] se presenta un abordaje de localización usando múltiples sensores de dispositivos móviles, dentro de ellos el campo magnético y el análisis de su interacción mediante el uso de filtros de partículas (FP).

El módulo de fingerprinting geomagnético utiliza mediciones del campo magnético. El magnetómetro 3D de un móvil mide el campo magnético en el sistema de tres coordenadas. Como el móvil puede ser orientado arbitrariamente en la mano del usuario, las mediciones se transforman al sistema de coordenadas horizontal normalizadas, los ángulos de orientación de dispositivos necesarios para la transformación se estiman por el uso de sensores inerciales (PDR) [10].

\section{DISEÑO DEL MODELO}

Para abordar el desarrollo de la investigación desde una perspectiva de experimentación cuantitativa, se desarrolló un estudio de caso que permitió replicar los resultados documentados por [9]. Se introduce una variable adicional, el comportamiento del campo magnético terrestre en interiores, para proponer un modelo más sofisticado de localización en interiores basado en las capacidades de procesamiento de los dispositivos móviles. La Figura 1 muestra el diagrama de flujo del abordaje metodológico.

\section{Entorno de estudio}

El entorno sobre el que se desarrolló el caso de estudio se constituye de una estructura civil de tipo habitacional, ubicada en la segunda planta de un edificio de cuatro plantas. Su área es de 56 metros cuadrados, constituidos por múltiples muros y columnas. Presencia de múltiples electrodomésticos 


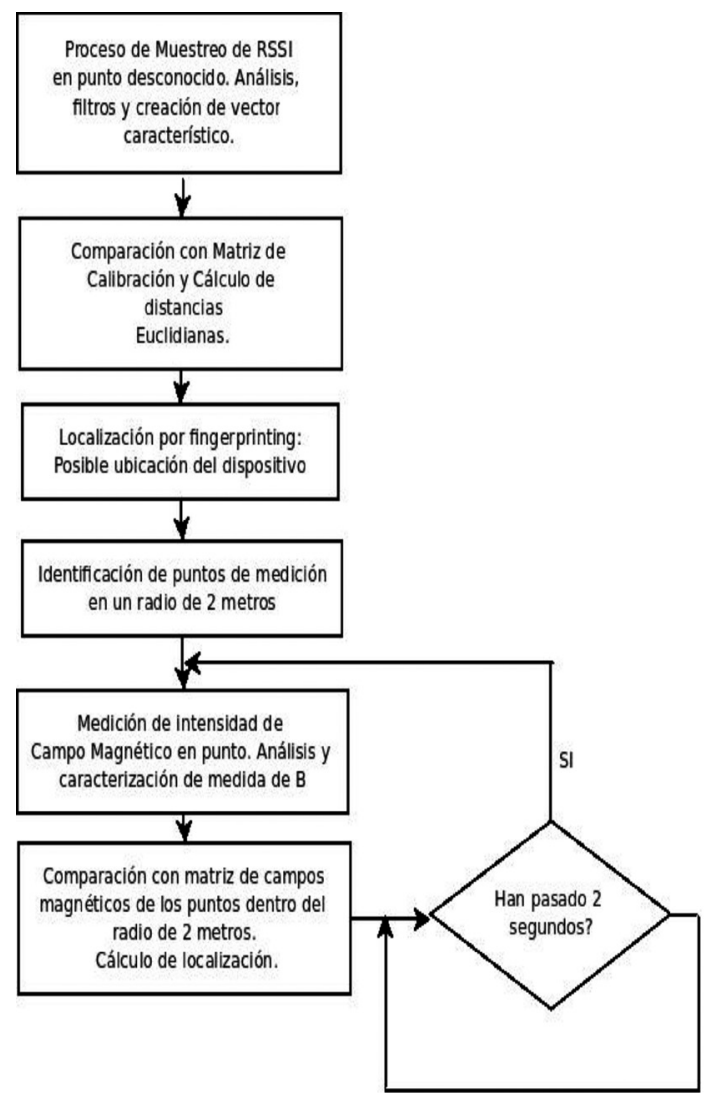

Figura 1. Diagrama de flujo del abordaje metodológico.

y múltiples redes WiFi propias y circundantes. Se trata de una estructura ubicada en la ciudad de Bogotá, Colombia, a una altura aproximada de $2.600 \mathrm{msnm}$.

Se replicaron los resultados obtenidos por [9] donde se aplican filtros estadísticos basados en la cantidad de mediciones de cada RSSI y el criterio de redes de bajo y alto porcentaje de aparición, donde las redes de bajo porcentaje de aparición corresponden a las redes distantes, que se perciben con baja potencia y comportamiento inestable. Por otro lado, las redes con porcentaje de aparición alto corresponden a las redes WiFi relativamente estables, cuya potencia de recepción no está en los umbrales bajos, y que presentan un escenario confiable de medición.

Luego se aplica el filtro de mediciones atípicas por boxplot y el filtro de Kalman para predicción de medidas estables.
La Figura 2 muestra el diagrama de flujos que sintetiza el proceso de calibración de fingerprinting por redes WiFi.

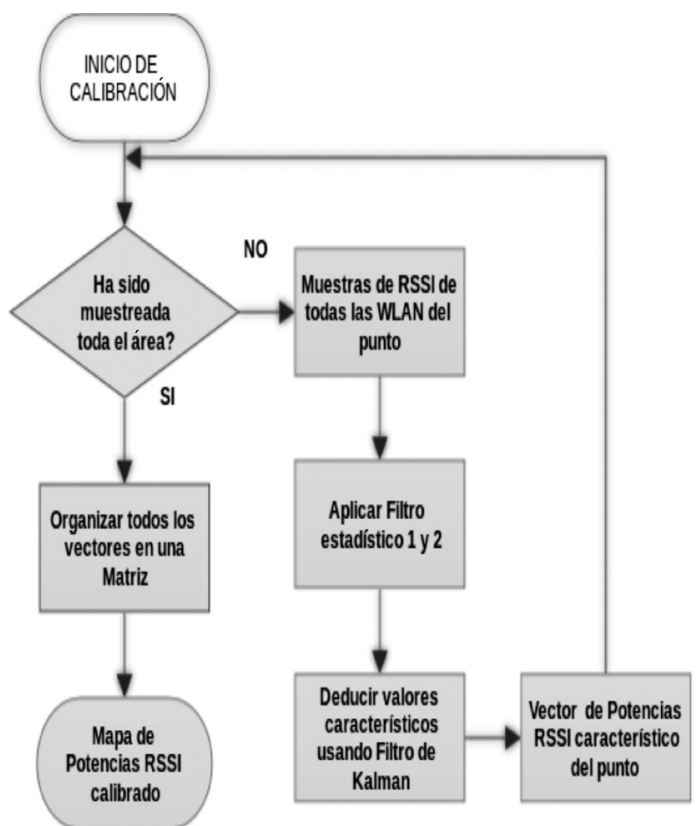

Figura 2. Diagrama de flujos de la etapa de calibración de RSSI para redes WiFi.

En la etapa de localización por fingerprinting, dado que se refiere al proceso de caracterizar las potencias RSSI de un punto particular desconocido, se aplica la rama derecha de la Figura 2 donde se logra construir el vector característico de dicho punto, lo que servirá para realizar un proceso de comparación con la matriz de potencias logradas en la calibración, para inferir la ubicación del punto desconocido.

El proceso de comparación se realiza por medio del cálculo de distancias euclidianas (d) dada por:

$$
d=\sqrt{\sum_{j=1}^{n}\left(P C_{i j}-P L_{j}\right)^{2}}
$$

Donde $j$ representa cada uno de los SSID muestreados en el punto a localizar, $P C$ es la potencia de calibración de cada SSID en el nodo $i$, y $P L$ es la potencia de cada SSID en el punto a localizar. Así se tendrán $\mathrm{m}$ distancias euclidianas, 
donde $m$ es la cantidad de nodos existentes. Las tres menores distancias euclidianas servirán para deducir la localización del dispositivo.

Es necesario anotar que la orientación de este trabajo consiste en que estos cálculos sean hechos por el procesador de un teléfono inteligente típico; así, entre mayor sea la cantidad $m$ de nodos, mayor será el costo computacional, y menor será el desempeño en el tiempo de respuesta.

La localización por fingerprinting implica una gran cantidad de datos por analizar. Debido a que la perspectiva es encontrar un modelo que pueda ser usado por un dispositivo móvil para localizarse en tiempo real, se decide abordar el estudio de una variable adicional, que implique un costo computacional mucho menor y pueda complementar la metodología de fingerprinting. Por tanto, y según lo analizado en el estado del arte, se decide abordar el estudio de comportamiento del campo magnético terrestre en interiores.

\section{Análisis de deformación del campo magnético terrestre}

Para las mediciones de campo magnético se usa un dispositivo móvil común con sensor magnetómetro. El magnetómetro puede captar la intensidad de campo magnético X, Y y Z como se aprecia en la Figura 3. Con estas medidas el magnetómetro puede calcular la intensidad total de campo magnético $B$ usando:

$$
B=\sqrt{B_{X}^{2}+B_{Y}^{2}+B_{Z}^{2}}
$$

Donde $B x, B y, y B z$ son las componentes de magnitud vectorial del campo magnético detectadas por el magnetómetro.

Debido a los movimientos naturales del dispositivo móvil, es conveniente usar la magnitud total del campo magnético dada por la ecuación (2) en vez de las magnitudes individuales de cada eje. Por tal razón las mediciones hechas solo contemplaron esta medida. Además el sistema operativo móvil hace autónomamente el cálculo de intensidad total del campo magnético.

Como análisis matemático se propone la realización de dos de los filtros usados en la metodología WiFi (filtro de medidas atípicas por boxplot y filtro de

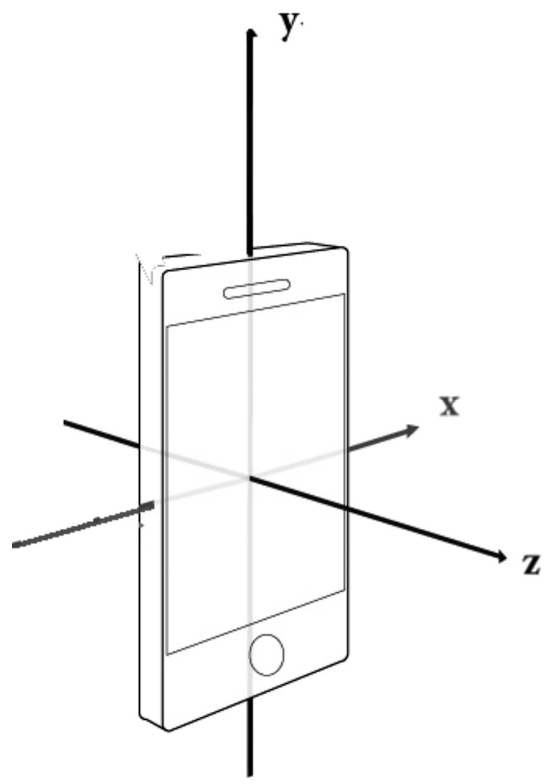

Figura 3. Magnetómetro en dispositivos móviles.

Kalman), con el fin de estabilizar las mediciones y caracterizar mejor el valor típico del campo magnético en un punto.

Se realizó análisis de boxplot a cada una de las muestras del campo magnético para identificar la estabilidad de las muestras y descartar medidas atípicas. Luego se aplica también el filtro de Kalman y se obtiene un valor único el campo magnético por punto.

En [11] se indica que las perturbaciones del campo magnético constituyen fuentes de información para la localización en espacio de interiores dado que indican con precisión la cercanía del equipo a fuentes de deformaciones como columnas de cableado, columnas con gran cantidad de material ferroso, equipos electrónicos, entre otros.

En las Figuras 4 y 5 se pueden evidenciar una anomalía de campo magnético, debido a ciertos tipos de materiales, esto se puede evidenciar en los cambios abruptos en la gráfica generada por el magnetómetro. La gráfica se obtuvo caminado linealmente por el espacio interior y manteniendo constante la captura de intensidad del campo magnético. Aquí el magnetómetro captura la presencia de la columna con cableado eléctrico, generando un pico en la gráfica, y el otro más bajo es causado por la ventana. 
uT

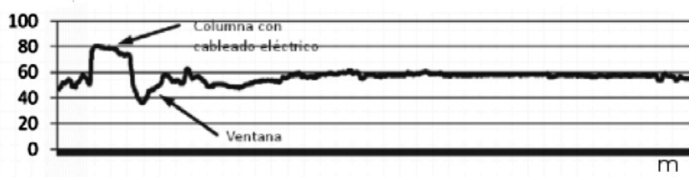

Figura 4. Evidencia de marcas magnéticas en el punto 1 .

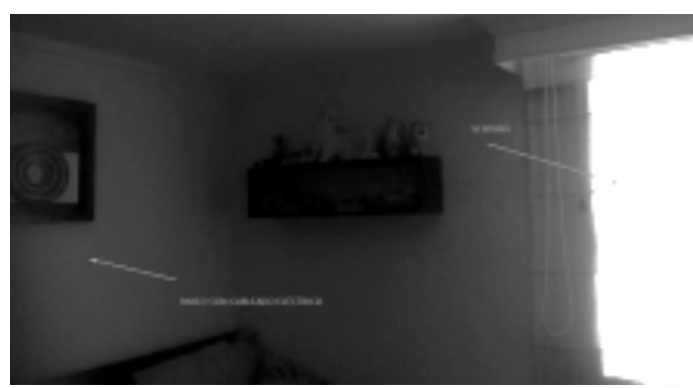

Figura 5. Espacio relativo a la Figura 4.

En la Figura 6 se puede evidenciar como el campo magnético generado por el televisor es detectado por el magnetómetro (pico de la gráfica), y se observa como un espacio libre también presenta características distinguibles en la intensidad de campo magnético.

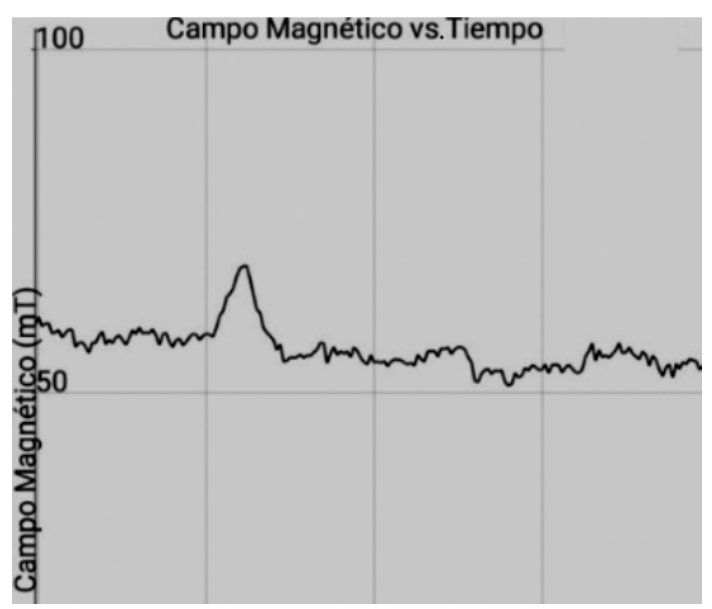

Figura 6. Evidencia de marcas magnéticas generadas por electrodomésticos.

Algunas variaciones del campo magnético dentro de los edificios se deben a la presencia de componentes como el hierro, cobalto o níquel y también se producen por estructuras de acero, sistemas de

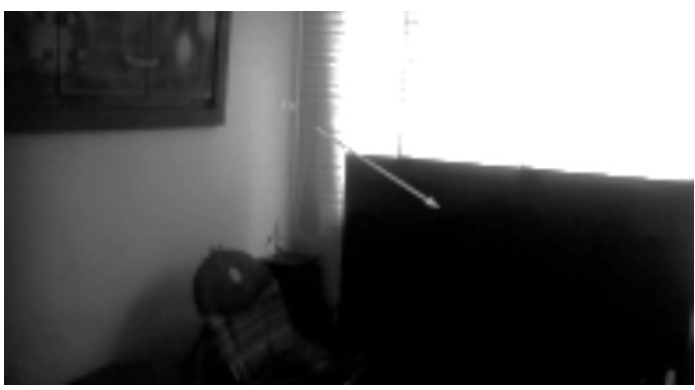

Figura 7. Espacio relativo a la Figura 6.

energía eléctrica y aparatos electrónicos [11]. Como se aprecia en esta serie de figuras, las variaciones pueden ser identificadas claramente, y salvo las anomalías causadas por electrodomésticos, el resto de anomalías asociadas a la estructura física del edificio son constantes y se mantienen en el tiempo. Así, es posible caracterizar claramente puntos de referencia que den información de la localización de un objeto.

Debido a que el análisis de campo magnético en cada punto permite tener un único valor característico, a diferencia de WiFi en el que se tiene un valor por cada SSID presente, es posible caracterizar fácilmente el espacio completo y reflejar eso en un mapa de calor. La Figura 8 muestra el caso de estudio donde se han aplicado los filtros y es posible tener una representación gráfica de un mapa de calor del campo magnético en un espacio de interiores.

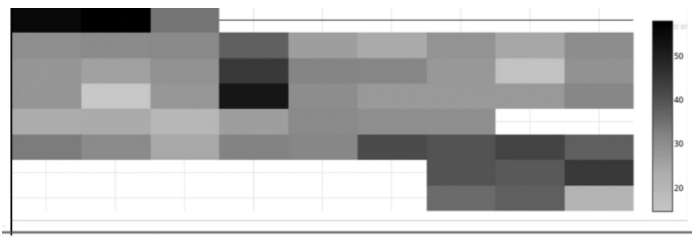

Figura 8. Mapa de calor estructura civil.

Entendiendo que en los colores más notorios es donde se evidenció la presencia del campo magnético con más intensidad, lo que se le puede llamar "marca magnética" y los colores más claros, el campo magnético con menor intensidad, lo que también representa una anomalía.

Esto quiere decir que puede ser determinado un vector de intensidades de campo magnético, que 
representan los valores típicos de este campo $B$ en cada uno de los puntos muestreados, y relacionado por la ecuación (3).

$$
V_{B}=\left\langle B_{1}, B_{2}, B_{3}, \ldots B_{n}\right\rangle
$$

Donde $V_{B}$ es el vector de campos magnéticos, y $n$ es la cantidad de puntos de medición. $V_{B}$ caracterizará el comportamiento del campo magnético en el espacio de interiores.

\section{Localización usando campo magnético}

En la etapa de localización, el dispositivo móvil realizará la medición del campo magnético del punto desconocido, realizará el tratamiento de datos pertinentes para hallar la medida característica del campo $B$ en ese punto. Es posible aplicar la técnica de cálculo de distancia euclidiana, con las medidas de intensidad de campo $B$, para hallar los puntos que aritméticamente sean más cercanos; sin embargo, ya que solo se está comparando un solo valor, la ecuación de distancia euclidiana para muestras de campo magnético $d_{B}$ se reduce a una simple diferencia, como se indica en la ecuación 4.

$$
d_{B} \sqrt{\left(B C_{j}-B L\right)^{2}=B C_{j}-B L}
$$

Donde $B C_{j}$ indica la intensidad de $B$ en cada uno de los puntos muestreados. $B L$ indica la intensidad de $B$ en el punto a localizar.
Sin embargo, dado que existe una sola variable $(B)$ la que se está usando como eje de las comparaciones, a diferencia del modelo de fingerprinting que tiene una cantidad de $\mathrm{M}$ variables, asociadas a las $M$ señales SSID presentes en el espacio; es necesario realizar una acotación más corta, y no medir la distancia euclidiana de los $B$, con todos los campos $B C_{j}$, sino con una porción menor ubicada en la zona que más tenga probabilidad de contener el dispositivo que está siendo localizado.

Debido a todo lo anterior, se desarrolla un modelo que se usa un cálculo inicial de localización por fingerprinting de redes WiFi para ubicar el dispositivo en una sección general del espacio, y luego se usa la localización por campos magnéticos para hacer mediciones más rápidas, hacer cálculos de distancia euclidiana de $B$ de solo los puntos más próximos al indicado por la técnica de fingerprinting, y además apoyarse de las marcas magnéticas, para realizar una localización rápida y ágil desde el propio dispositivo móvil.

\section{RESULTADOS Y DISCUSIÓN}

La Tabla 1 muestra los resultados replicados en [9]. Se calcula la distancia de error, entendida como la distancia lineal entre la posición real del dispositivo móvil, y la posición calculada con las dos metodologías mencionadas en [9]. En la mayoría de muestras, la localización usando los nuevos filtros propuestos es mucho mejor, porque la distancia de error es notoriamente menor.

Tabla 1. Resultados de localización usando métodos de fingerprinting de WiFi [9].

\begin{tabular}{|c|c|c|c|c|c|c|c|c|}
\cline { 2 - 9 } \multicolumn{1}{c|}{} & \multicolumn{2}{|c|}{ Posición real } & \multicolumn{2}{c|}{$\begin{array}{c}\text { Posición calculada con } \\
\text { desviación estándar }\end{array}$} & $\begin{array}{c}\text { Distancia de } \\
\text { error (de) }\end{array}$ & \multicolumn{2}{c|}{$\begin{array}{c}\text { Posición calculada con } \\
\text { boxplot y filtro Kalman }\end{array}$} & $\begin{array}{c}\text { Distancia de } \\
\text { error (de) }\end{array}$ \\
\hline Ubicación & $\mathbf{X}$ & $\mathbf{Y}$ & $\mathbf{X}$ & $\mathbf{Y}$ & Metros & $\mathbf{X}$ & $\mathbf{Y}$ & Metros \\
\hline 1 & 4,5 & 5,2 & 3,43 & 5,99 & 1,33 & 4,44 & 4,96 & 0,24 \\
\hline 2 & 5,5 & 2,5 & 6,17 & 2,68 & 0,70 & 5,66 & 2,92 & 0,45 \\
\hline 3 & 7 & 3,7 & 5,71 & 3,66 & 1,29 & 6,09 & 3,34 & 0,98 \\
\hline 4 & 6,5 & 5,7 & 5,41 & 3,28 & 2,66 & 7,01 & 5,35 & 0,62 \\
\hline 5 & 8 & 4,7 & 7,32 & 4,98 & 0,74 & 7,01 & 5,35 & 1,19 \\
\hline 6 & 8 & 2,5 & 7,01 & 4,23 & 1,99 & 8,72 & 2,85 & 0,80 \\
\hline 7 & 9,5 & 5,2 & 7,30 & 5,00 & 2,21 & 9,33 & 4,04 & 1,17 \\
\hline 8 & 10,5 & 4,7 & 10,00 & 5,69 & 1,11 & 9,04 & 3,53 & 1,87 \\
\hline 9 & 10,5 & 2,2 & 10,04 & 1,24 & 1,06 & 10,36 & 1,69 & 0,52 \\
\hline 10 & 9 & 1,7 & 9,66 & 1,06 & 0,92 & 10,22 & 1,65 & 1,22 \\
\hline
\end{tabular}


De la Tabla 1 se deduce que la metodología propuesta para el mejoramiento del fingerprinting tiene una exactitud en la localización, representada por la distancia de error, de entre $0,2 \mathrm{~m}$ y $1,87 \mathrm{~m}$, y en promedio este error está por debajo de $1 \mathrm{~m}$.

Aún con los buenos datos obtenidos y la reducción en el error de localización, no es eficiente realizar este tipo de cálculos en dispositivos móviles debido a la gran cantidad de datos a analizar y el gran costo de procesamiento que esto implica en un dispositivo de esas características.

En la Tabla 2 se resume el tiempo tomado en el procesamiento de ciertas rutinas basado en un dispositivo móvil con las siguientes características:

Sistema operativo Android 4.2

Procesador Quad-Core $1.5 \mathrm{GHz}$

Memoria RAM 1 GB

Tabla 2. Tiempo de procesamiento con dispositivo móvil y método de fingerprinting.

\begin{tabular}{|l|c|}
\hline \multicolumn{1}{|c|}{ Proceso } & \multicolumn{1}{|c|}{$\begin{array}{c}\text { Tiempo } \\
\text { aproximado para } \\
\text { procesamiento } \\
\text { (segundos) }\end{array}$} \\
\hline $\begin{array}{l}\text { Análisis de frecuencia de } \\
\text { aparición de 56 SSID presentes } \\
\text { en un punto. }\end{array}$ & 5 \\
\hline $\begin{array}{l}\text { Análisis de boxplot de 40 SSID } \\
\text { presentes en un punto. }\end{array}$ & 15 \\
\hline $\begin{array}{l}\text { Cálculo de valor característico } \\
\text { por filtro de Kalman de 40 } \\
\text { SSID presentes en un punto. }\end{array}$ & 50 \\
\hline Localización & 50 \\
\hline
\end{tabular}

Tabla 3. Tiempo de procesamiento con dispositivo móvil y método de huellas de CM.

\begin{tabular}{|l|c|}
\hline \multicolumn{1}{|c|}{ Actividad } & \multicolumn{1}{c|}{$\begin{array}{c}\text { Tiempo } \\
\text { aproximado para } \\
\text { procesamiento } \\
\text { (segundos) }\end{array}$} \\
\hline $\begin{array}{l}\text { Análisis de boxplot de una } \\
\text { medida de campo magnético. }\end{array}$ & $<1$ \\
\hline $\begin{array}{l}\text { Cálculo de valor característico } \\
\text { por filtro de Kalman de una } \\
\text { medida de campo magnético. }\end{array}$ & 1 \\
\hline
\end{tabular}

La gran cantidad de datos que deben ser procesados hacen que no sea factible el cálculo de localización en tiempo real, basado exclusivamente en el estudio de las potencias de RSSI.

Se realizan los cálculos de localización usando las medidas de intensidad de campo magnético porque la medición de campo magnético solo precisa de una única medida, y además se restringen a los puntos ubicados a dos metros alrededor del posible punto de localización dado por la metodología de fingerprinting (en concordancia con la Figura 1), el costo computacional para su procesamiento es significativamente más bajo. La Tabla 3 muestra los tiempos tomados por el mismo dispositivo móvil usado en el estudio de caso de potencias RSSI.

Aplicando el modelo propuesto en la Figura 1, donde se hace un análisis del campo magnético basado en los puntos de los dos metros alrededor del punto calculado por el fingerprinting del WiFi, se obtienen los resultados de las Tablas 4 y 5 .

Como se pueden observar en las Tablas 4 y 5, la metodología complementaria propuesta usando la localización por fingerprinting de redes WiFi y el análisis de deformación del campo magnético, tiene un error superior al error presentado por la metodología solamente basada en análisis de redes WiFi en [9]. Sin embargo, las Tablas 2 y 3 muestran la gran diferencia en tiempos de procesamiento obtenidas mediante ambas metodologías.

Se puede decir que aunque la metodología complementaria entre localización por redes WiFi y análisis del campo magnético, tiene un error superior, su ventaja en tiempos de procesamiento la hace una alternativa viable de ser usada en entornos donde todo el procesamiento depende del dispositivo móvil.

\section{CONCLUSIONES}

La metodología de análisis de potencias RSSI permite identificar rápidamente la habitación donde se ubica el dispositivo, el análisis de deformación del campo magnético terrestre permite identificar el lugar dentro de esa habitación de forma ágil, y repetitiva. Así, la metodología del estudio y muestreo de campo magnético es una variable complementaria para la metodología basada en radiación de redes WiFi. 
Tabla 4. Resultados obtenidos con el métedo propuesto.

\begin{tabular}{|c|c|c|c|c|c|c|c|c|}
\cline { 2 - 9 } & \multicolumn{2}{|c|}{$\begin{array}{c}\text { Posición } \\
\text { real }\end{array}$} & $\begin{array}{c}\text { Posición } \\
\text { calculada con } \\
\text { boxplot y } \\
\text { filtro Kalman }\end{array}$ & \multicolumn{2}{c|}{$\begin{array}{c}\text { Cálculo de posición } \\
\text { usando campo } \\
\text { magnético de puntos } \\
\text { con 2 metros alrededor }\end{array}$} & $\begin{array}{c}\text { Distancia de error } \\
\text { (de) con boxplot y } \\
\text { filtro Kalman }\end{array}$ & $\begin{array}{c}\text { Distancia de error } \\
\text { (de) con campo } \\
\text { magnético }\end{array}$ \\
\hline Ubicación & $\mathbf{X}$ & $\mathbf{Y}$ & $\mathbf{X}$ & $\mathbf{Y}$ & $\mathbf{X}$ & $\mathbf{Y}$ & Metros & Metros \\
\hline 1 & 4,5 & 5,2 & 4,44 & 4,96 & 4,02 & 4,73 & 0,24 & 0,67 \\
\hline 2 & 5,5 & 2,5 & 5,66 & 2,92 & 5,30 & 3,21 & 0,45 & 0,74 \\
\hline 3 & 7 & 3,7 & 7,01 & 3,34 & 5,30 & 4,50 & 0,98 & 1,88 \\
\hline 4 & 6,5 & 5,7 & 7,01 & 5,35 & 7,40 & 5,60 & 0,62 & 0,91 \\
\hline 5 & 8 & 4,7 & 8,72 & 2,85 & 7,30 & 5,10 & 1,19 & 0,81 \\
\hline 6 & 8 & 2,5 & 7,01 & 4,04 & 9,10 & 2,60 & 0,80 & 1,10 \\
\hline 7 & 9,5 & 5,2 & 9,33 & 3,53 & 9,10 & 4,43 & 1,17 & 0,87 \\
\hline 8 & 10,5 & 4,7 & 9,04 & 1,69 & 9,50 & 3,80 & 1,87 & 1,35 \\
\hline 9 & 10,5 & 2,2 & 10,36 & 1,24 & 8,80 & 1,70 & 0,52 & 1,77 \\
\hline 10 & 9 & 1,7 & 10,22 & 1,65 & 10,30 & 1,80 & 1,22 & 1,30 \\
\hline
\end{tabular}

Tabla 5. Análisis de la Tabla 4.

\begin{tabular}{|l|c|c|}
\hline & $\begin{array}{c}\text { Distancia de } \\
\text { error (de) con } \\
\text { boxplot y filtro K }\end{array}$ & $\begin{array}{c}\text { Distancia de } \\
\text { error (de) con } \\
\text { campo magnético }\end{array}$ \\
\hline Desv est. & 0,48 & 0,43 \\
\hline Máx. & $1,87 \mathrm{~m}$ & $1,88 \mathrm{~m}$ \\
\hline Mín. & $0,24 \mathrm{~m}$ & $0,67 \mathrm{~m}$ \\
\hline Promedio & $0,91 \mathrm{~m}$ & $1,14 \mathrm{~m}$ \\
\hline
\end{tabular}

Mediante el caso de estudio se corrobora la validez del análisis de potencias de RSSI mediante las técnicas de fingerprinting, para localizar dispositivos en interiores; sin embargo, se evidencia que requiere el procesamiento de una gran cantidad de datos, que hace que no sea viable para su uso autónomo en dispositivos móviles. Aunque existe la posibilidad de delegar esa función a servidores externos, no es la intención de esta aproximación.

Se evidenció que las deformaciones de campo magnético terrestre debido a la presencia de electrodomésticos, columnas con materiales ferrosos y cableados eléctricos generan marcas magnéticas que permiten caracterizar un espacio completo y brindar información de localización por medio de un mapa de calor.

Se propone como trabajo futuro un estudio sobre otras variables como las proporcionadas por los sensores inerciales, para poder calcular desplazamientos y predecir movimientos.

\section{REFERENCIAS}

[1] K. Nuaimi, H. Kamel. "A Survey of Indoor Positioning Systems and Algorithms". International Conference on Innovations in Information Technology (IIT). IEEE. DOI 10.1109/INNOVATIONS.2011.5893813. Abu Dhabi, Emiratos Arabes. Abril 2011.

[2] K. Kaemarungsi, P. Krishnamurthy. "Modeling of Indoor Positioning Systems Based on Location Fingerprinting". INFOCOM 2004. Twenty-third AnnualJoint Conference of the IEEE Computer and Communications Societies. Vol. 2. DOI: 10.1109/INFCOM.2004.1356988. IEEE. Hong Kong, República Popular China. Marzo 2004.

[3] R. Zekavat et al. "Handbook of Position Location: Theory, Practice and Advances". Wiley-IEEE Press. Ed 1. Vol. 1, pp. 28-33. ISBN: 978-0-470-94342-7. 2011.

[4] S. Souvik, L. Jeongkeun, K. Kyu-han, C. Paul. "Avoiding Multipath to Revive Inbuilding WiFi Localization”. ACM MobiSys. Taipei, Taiwan. Junio 2013.

[5] P. Bahl, V. Padmanabhan. "RADAR: an in-building RF-based user location and tracking system”. Proceedings IEEE INFOCOM 2000. Conference on Computer Communications. Nineteenth Annual Joint Conference of the IEEE Computer and Communications Societies (Cat. 
$\mathrm{N}^{\circ}$ 00CH37064), 2, 775-784. doi:10.1109/ INFCOM.2000.832252. Tel Aviv, Isreael. Marzo 2000.

[6] A. Moura, C. Ribeiro. A. Costa. "WBLS: A signal presence-based Wi-Fi localisation system for mobile devices in smart environments". International Journal of Knowledge-Based Intelligent Engineering Systems. Vol. 13, pp. 5-18. DOI: 10.3233/ KES-2009-0169. 2009.

[7] L. Descamps-Vila, A. Pérez-Navarro and J. Conesa. "RSS and sensor fusion algorithms for indoor location systems on Smartphones". Connecting a Digital Europe Through Location and Place. Lecture Notes in Geoinformation and Cartography. Ed Springer, pp. 197-214. DOI: $10.1007 / 978-$ 3-319-03611-3_12. 2014.

[8] A. Teuber. "WLAN Indoor Positioning Based on Euclidean Distances and Fuzzy Logic". Institute of Geodesy and Navigation, University FAF. 3rd Workshop On Positioning,
Navigation And Communication. Munich, Alemania. 2006.

[9] C. Gómez. "Localización en espacios interiores de dispositivos móviles por medio de tecnologías WiFi, base para el desarrollo de aplicaciones sociales". Encuentro Internacional de Educación en Ingeniería EIEI2015. Cartagena, Colombia. 2015.

[10] B. Gennady. "Accurate and Reliable RealTime Indoor Positioning on Commercial Smartphones". Proceedings of the 2014 International Conference on Indoor Positioning and Indoor Navigation (IPIN), IEEE Xplore. IEEE Catalog Number: CFP1409J-ART. ISBN: 978-1-4673-8054-6. 2014.

[11] P. Kalyan. "Magnetic-fields-based indoor localization using Smartphones". ACM Transactions on Intelligent Systems and Technology (TIST). Vol. 4 Issue 4. Article $\mathrm{N}^{\circ}$ 73. DOI: $10.1145 / 2508037.2508054$. Septiembre 2013. 